\title{
Spontaneous Retropharyngeal and Mediastinal Emphysema
}

\author{
Do-Yeon Cho ${ }^{1} \cdot$ Geoffrey P. Aaron ${ }^{1} \cdot$ Kimberly G. Shepard ${ }^{2}$ \\ ${ }^{1}$ Division of Otolaryngology-Head and Neck Surgery, Department of Surgery, University of Alabama at Birmingham, Birmingham, AL; \\ ${ }^{2}$ Division of Otolaryngology-Head and Neck Surgery, Santa Clara Valley Medical Center, San Jose, CA, USA
}

A 14-year-old girl with no significant medical history presented at Emergency Department with sore throat and odynophagia after one episode of nonviolent coughing. She denied any respiratory distress, voice change, foreign body ingestion, retching, substance abuse, dental procedures, or trauma. She was afebrile with normal oxygen saturation and physical examination including the head and neck was unremarkable with the exception of bilateral neck crepitus without tenderness on palpation. Fiberoptic laryngoscopy revealed a patent laryngeal airway with normal vocal fold movement. Lateral neck $\mathrm{X}$-ray demonstrated a linear air-column in the retropharyngeal space and computed tomography confirmed emphysema involving the retropharyngeal space and mediastinum with no evidence of fluid collection or abscess formation. Spontaneous retropharyngeal and mediastinal emphysema are clinical entities where free air is present within the confines of retropharyngeal space and mediastinum without obvious cause. It is benign and self-limited in nature and allows for conservative management. This case is presented with a review of literature.

Keywords. Mediastinal Emphysema; Subcutaneous Emphysema; Mediastinitis

\section{INTRODUCTION}

Spontaneous retropharyngeal and mediastinal emphysema (SRE and SME) are rare clinical entities where free air is present within the confines of retropharyngeal space and mediastinum respectively, without apparent cause. Excluding a history of surgery and trauma, the leading cause is bronchial asthma, followed by physical exertion, forced swallowing, or any behavior producing instant positive pressure in the upper airway. Examples include as Valsalva maneuver, shouting or coughing [1-3]. Clinical symptoms vary depending on the involved confined space, from mild sore throat to acute airway obstruction $[1,4]$. It is crucial for otolaryngologists to be aware of the clinical presentation and differential diagnosis associated with SRE, as the air in the retropharyngeal space can develop into mediastinitis and a re-

\footnotetext{
- Received October 14, 2014

Accepted March 11, 2015

- Corresponding author: Kimberly G. Shepard

Division of Otolaryngology-Head and Neck Surgery, Santa Clara Valley

Medical Center, 751 S Bascom Ave, San Jose, CA, USA

Tel: +1-408-885-6947, Fax: +1-408-885-3589

E-mail: Kimberly.Shepard@hhs.sccgov.org
}

sulting poor outcome [4]. Therefore, prompt evaluation is essential to prevent such disastrous consequences. We would like to describe one case of SRE and SME from a young healthy adolescent female who presented with sore throat.

\section{CASE REPORT}

A 14-year-old girl, with no significant medical history, presented to the Emergency Department with sore throat and odynophagia after one episode of nonviolent coughing, prior to the admission. She denied shortness of breath, voice changes, retrosternal chest pain, or febrile sensation. There was no recent history of upper respiratory infection, foreign body ingestion, retching, substance abuse, dental procedures or trauma. Vital signs on presentation were normal without fever or stridor and physical examination including head and neck region was unremarkable except for bilateral neck crepitus without tenderness, on palpation. Flexible fiberoptic nasopharyngolaryngoscopy revealed no bulging of posterior pharyngeal wall and a patent laryngeal airway with normal vocal fold movement. Radiological evaluation was performed. Lateral radiography of neck demon-

Copyright @ 2016 by Korean Society of Otorhinolaryngology-Head and Neck Surgery.

This is an open-access article distributed under the terms of the Creative Commons Attribution Non-Commercial License (http://creativecommons.org/licenses/by-nc/4.0)

which permits unrestricted non-commercial use, distribution, and reproduction in any medium, provided the original work is properly cited. 


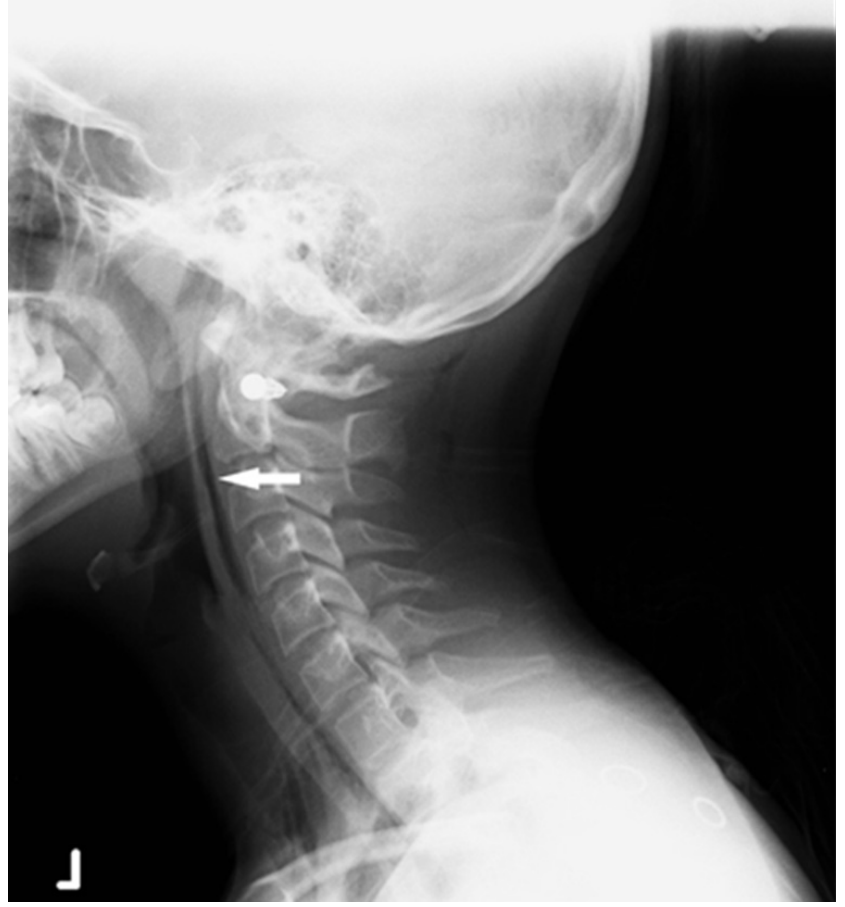

Fig. 1. Lateral radiography of neck demonstrated a linear air-column (arrow) in the retropharyngeal space. Note the metal shadow between $\mathrm{C} 1$ and $\mathrm{C} 2$ spine is patient's ear ring.
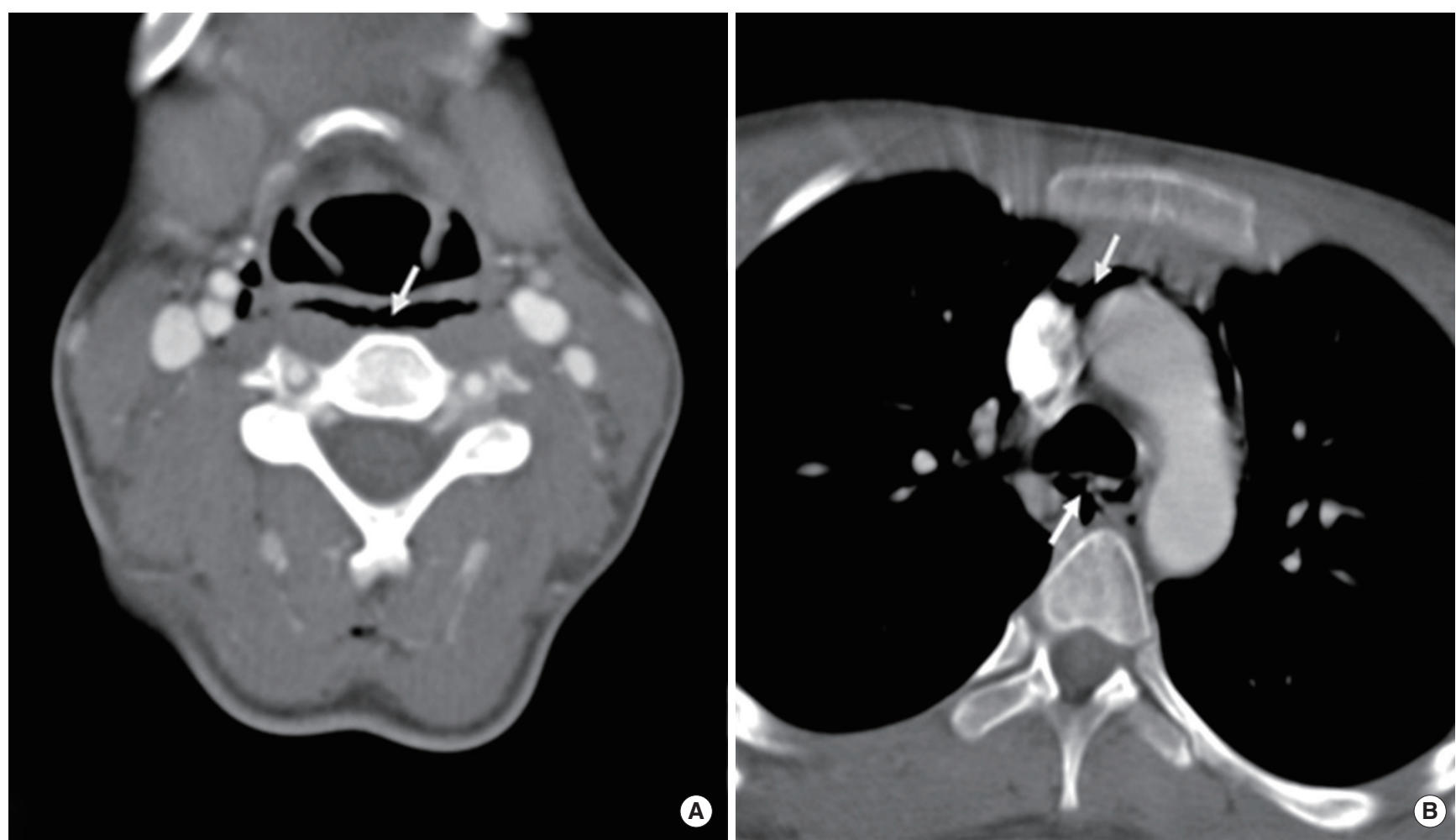

Fig. 2. Computed tomography confirmed extensive cervical emphysema involving the retropharyngeal space (A: arrow) and mediastinum (B: arrows) with no evidence of fluid collection or abscess formation. (A) Axial image at the level of hypopharynx. (B) Axial image at the level of mediastinum. strated a linear air-column in the retropharyngeal space (Fig. 1). Computed tomography confirmed extensive cervical emphysema involving the retropharyngeal space and mediastinum with no evidence of fluid collection or abscess formation (Fig. 2). Patient was diagnosed with SRE and SME and admitted to pediatric intensive care unit for close observation. She was managed conservatively with bed rest, nothing by mouth, supplemental oxygen via nasal cannula, intravenous antibiotics (clindamycin $20 \mathrm{mg} / \mathrm{kg} /$ day) and intravenous hydration. Patient's sore throat gradually resolved and near-complete resolution of linear air column in the retropharyngeal space was confirmed with radiography of lateral neck on the second hospital day (Fig. 3). Patient was discharged to home on the third hospital day, with 10day course of oral clindamycin and instructions to abstain from strenuous activities. Since then, the patient has not had any further recurrence.

\section{DISCUSSION}

SRE and SME are uncommon, benign, self-limited, disorders that usually occur in young adults without any apparent precipitating factor or disease [4,5]. Hamman is credited in 1939 with identifying one of the first cases of SME [1]. Macklin and Mack- 


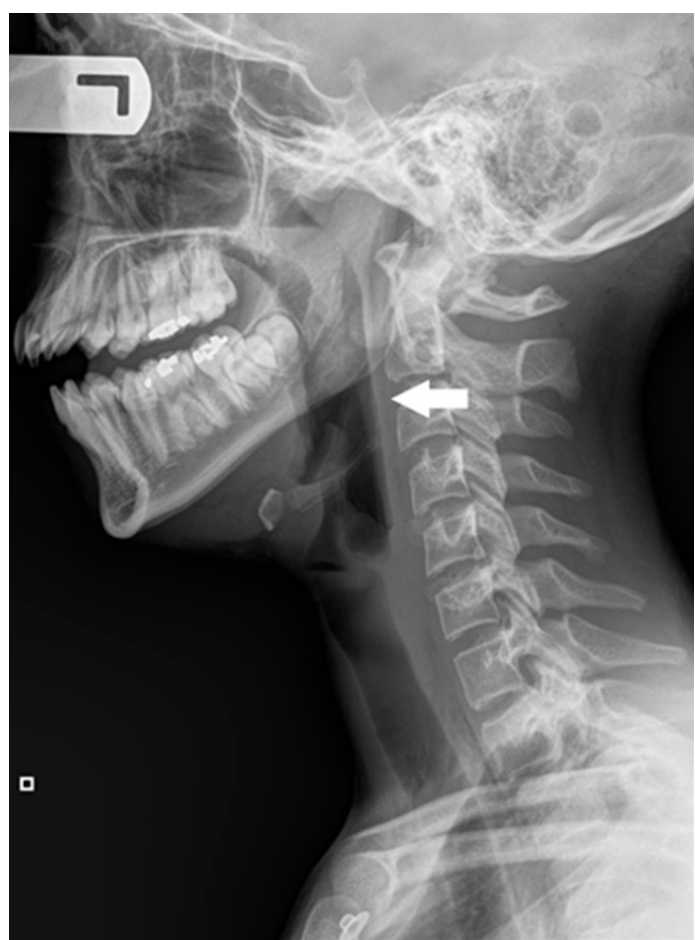

Fig. 3. Near-complete resolution of linear air column (arrow) in the retropharyngeal space was confirmed with radiography of lateral neck on the second hospital day.

lin [2] went further and described the pathophysiology of SME in 1944 based on the results of their animal study, and concluded that it occurred from barotrauma and alveolar structure disruption caused by intra-alveolar space hypertension associated with bronchial obstruction and led to dissection of air up the bronchovascular sheath into the superficial cervical and retroperitoneal spaces.

Since then, only a handful of case reports and small retrospective case series have been published discussing this clinical presentation. A 2005 case review of 18 patients by Newcomb and Clarke [3] estimated that 1 in 29,670 Emergency Department visits were due to a diagnosis of SPM. The majority of these patients were young males who had a history of asthma, smoking tobacco, or illicit drugs use. None of the patients in this series had serious acute issues in relation to the SPM and all resolved without long term complications. Only one patient required invasive therapy by means of a chest tube placement. In many of the case reports and series of SRE and SME, related symptoms were improved with conservative measures. In 2004, Smith and Hsu [4] presented 4 patients who all had developed SRE and SME. All patients improved without any significant intervention. Despite most cases resulting in a benign course, in some instances, patients can develop more significant airway obstruction. Skogvoll et al. [5] reported a two and half year old boy who developed paroxysmal coughing spells from adenovirus. He was noted to have retropharyngeal emphysema and af- ter several days of observation and conservative therapy he developed significant airway obstruction, requiring intubation and chest tube placement. He eventually extubated without issue and had no long-term complications.

Occasionally, patients may present with isolated head and neck complaints without chest symptoms, resulting in otolaryngology consultation rather than pulmonary or thoracic referral, similar to the patient presented in this case report. These patients usually have vague atypical histories, rendering the diagnosis more difficult. Therefore, otolaryngologists should be aware of this entity and treatment. Smith and Hsu [4] recommended the following management scheme: (1) a thorough history and physical examination; (2) flexible laryngoscopy to evaluate the airway; and (3) proper imaging studies that may include neck radiograph, chest radiograph, computed tomography scan of neck and barium swallow to exclude the esophageal perforation. The benign and self-limited nature of SRE and SME allows for conservative management.

It is also important to recognize that despite the fact patients typically do very well without invasive or aggressive therapy, these patients can also worsen and decompensate. Previous case reports have indicated patients can develop acute airway obstruction $[5,6]$. One serious concern is development of mediastinitis following air contamination of the deep tissues, which can result in catastrophic outcomes in these patients. Therefore we treated our patient with short course of antibiotics for prophylaxis. Though mediastinitis should be ruled out with the abovementioned workup algorithm, the risk of delayed presentation still exists. It is recommended that upon discharge, patients with SRE and SME receive extensive instructions to return to the Emergency Department for further work-up if they develop any signs concerning for mediastinitis. This would include worsening dyspnea, chest pain, abdominal pain, or signs and symptoms related to shock [7].

In summary, we report a case of a young female with very mild symptomatology, including sore throat and odynophagia following a coughing episode, who was found to have SRE and SME on further workup. Typically this is a relatively benign, selflimited disease process. We advocate a thorough work-up with history and physical, laryngeal exam, and appropriate radiographic imaging. Therapy is typically conservative with pain management, oxygen, and bronchodilators. It is important to educate the patient on when to return for further evaluation.

\section{CONFLICT OF INTEREST}

No potential conflict of interest relevant to this article was reported. 


\section{REFERENCES}

1. Hamman L. Spontaneous mediastinal emphysema. Bull Johns Hopkins Hosp. 1939;64:1-21.

2. Macklin MT, Macklin CC. Malignant interstitial emphysema of the lungs and mediastinum as an important occult complication in many respiratory diseases and other conditions: an interpretation of the clinical literature in the light in the light of laboratory experiment. Medicine. 1944 Dec;23(4):281-358.

3. Newcomb AE, Clarke CP. Spontaneous pneumomediastinum: a benign curiosity or a significant problem? Chest. 2005 Nov;128(5):3298302.

4. Smith JL 2nd, Hsu JM. Spontaneous pneumomediastinum present- ing with retropharyngeal emphysema. Am J Otolaryngol. 2004 JulAug;25(4):290-4.

5. Skogvoll E, Grammeltvedt AT, Aadahl P, Mostad U, Slordahl S. Lifethreatening upper airway obstruction in a child caused by retropharyngeal emphysema. Acta Anaesthesiol Scand. 2001 Mar;45(3):3935.

6. Lee CC, Chen TJ, Wu YH, Tsai KC, Yuan A. Spontaneous retropharyngeal emphysema and pneumomediastinum presented with signs of acute upper airway obstruction. Am J Emerg Med. 2005 May; 23(3):402-4.

7. Brauer RB, Liebermann-Meffert D, Stein HJ, Bartels H, Siewert JR. Boerhaave's syndrome: analysis of the literature and report of 18 new cases. Dis Esophagus. 1997 Jan;10(1):64-8. 